\title{
How close are we to finding noninvasive markers for upper Gl tract cancer?
}
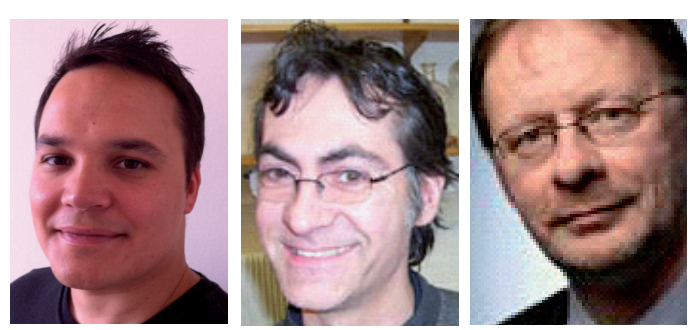

“...results highlight clearly the apparent redundancy of

biomarkers between

different disease processes..."

Richard JE Skipworth', Holger Husi' \& James A Ross ${ }^{\dagger 1}$

'Tissue Injury \& Repair Group, School of Clinical Sciences \& Community Health, University of Edinburgh.

1st Floor Chancellors Building, 49 Little France Crescent, Edinburgh, EH16 4SB, UK

†Author for correspondence: Tel..: +44 1312426520 - j.a.ross@ed.ac.uk

Cancer of the upper GI tract (i.e., esophagus, stomach and duodenum) represents a significant global problem. Gastric cancer is the fourth most common cancer worldwide, whereas esophageal adenocarcinoma has the fastest growing incidence rate of any tumor in the Western world, through its potential linkage to gastro-esophageal reflux and the premalignant condition of Barrett's esophagus. By comparison, duodenal cancer is relatively rare, and is mainly associated with certain hereditary syndromes. However, the prognosis of all of these tumour types is poor, with most studies reporting 5-year survival rates of approximately $8-15 \%$ [101]. Thus, the identification of noninvasive biomarkers for these tumor types represents a clear unmet clinical need. But how close are we to using such biomarkers in clinical practice?

\section{"...the next hurdle will be}

to develop a simple, clinical method of fingerprint assay that is sensitive, reproducible and cost effective (e.g., a urine dipstick test), but this ease of technology currently remains elusive."

When considering biomarker discovery, the chief goal is usually biomarkers for diagnosis. However, biomarkers indicating tumor sensitivity to treatment and patient prognosis would also be valuable tools, particularly during the development of clinical trials of novel treatments. Identified biomarkers may even represent therapeutic targets in their own right. However, biomarker discovery is not an easy challenge. Many recent studies of biomarker identification in upper GI tract cancer (our own included) have relied on complex technology (e.g., SELDI-TOF [1] and MALDI-TOF [2] mass spectrometry [MS]) in association with bioinformatic analysis, both of which may be time intensive, expensive, and require significant expertise. Recent recommendations for biomarker discovery have supported the use of robust statistical techniques and the verification of marker identification in independent test sets [3]. However, sophisticated methodologies are required for more than scientific accuracy. Earlier attempts at biomarker discovery targeted single markers. However, we now know that such a strategy is flawed, as many individual biomarkers are not specific to a single tumor type or histological subtype. Therefore, intricate, 'multiple biomarker' approaches are required to generate 'fingerprints', profiles of biomarkers that adequately describe the disease process in question. These multiple approaches offer more than just a technical challenge for the researcher. The wide range of existing technologies also means that the researcher must make additional decisions regarding both the platform and output of his or her study. The research platforms available include genomics, proteomics, metabolomics and, in tissue samples, transcriptomics also. Each platform offers different assays with relative advantages and disadvantages. However, at least two of these options might have the additional disadvantage that they do not offer any insights into either disease mechanism or potential drug targets.

A second decision facing the researcher who has elected to use a noninvasive technique for biomarker discovery is the choice of biofluid to be analyzed: plasma/serum, urine, saliva, sputum, feces or even gas (breath). Different

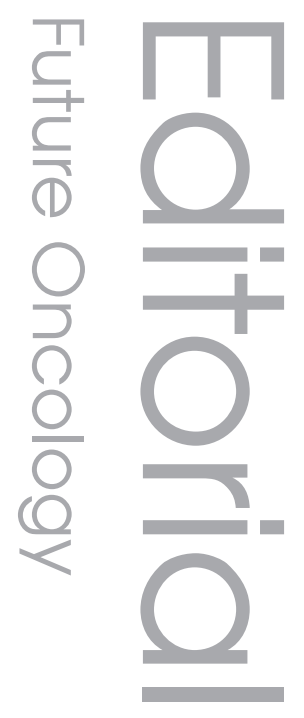

\section{Keywords}

- biomarker = cachexia

- diagnosis = esophageal cancer $=$ gastric cancer

- mass spectrometry = urine

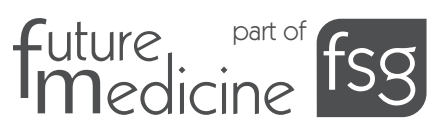


biofluids offer different possibilities for biomarker composition, ease of attainment, sample volume, and storage. In our own studies, we have used urine as the biofluid of choice within the context of a MS proteomics platform [1,2]. This is because urine is easy to sample; the patient can essentially provide an unlimited supply; and it offers a representative sample of system-wide circulation without the complexity of the proteome of plasma or serum. Heterogeneity in population genetics may mean that biofluids with a simple composition are more able to offer robust biomarker identification compared with more complex substrates. The urinary proteome contains approximately 3000 species [4], a third of which are classical circulatory plasma proteins that still occur in urine despite their retention to a large extent during the glomerular filtration process. Urine is relatively stable on storage and can be stabilized further with additives, whereas the proteome of other biofluids, such as serum, may be altered or degraded by endogenous proteases [1]

"As methods of radiological
imaging (including 'virtual'
endoscopy and functional imaging)
become more sensitive and
less toxic, it is not inconceivable that the
ultimate noninvasive method of all
cancer diagnosis will soon be a
whole-body scan."

Some authors have taken a more rigorous pathway to their choice of biofluid. Alterovitz et al. argued that the tissue being investigated should influence the choice of the biofluid [5]. As most current approaches to biomarker discovery analyze tissue and fluid separately, there is no conception of the 'functional distance' between the two. Furthermore, most proteomic studies rely simply on protein expression overlap between the tissue and the biofluid, but do not consider the potential for protein-protein interactions. By conceptualizing the tissue as a transmitter of information, the biofluid sampled by the researcher as a receiver of information, and the intervening tissue-biofluid relationship as an information channel through which data can be passed (but also lost [e.g., via renal filtration] or contaminated by other sources), the authors used information theory to conclude that the best biofluids in which to search for stomach-related biomarkers are plasma and, interestingly, saliva [5].
In our own attempts to identify diagnostic biomarkers of upper GI tract cancer, we used SELDI-TOF MS to analyze urine specimens from cancer patients and healthy controls to establish a potential biomarker fingerprint for the weak cation exchanger CM10 chip surface [1]. Blind testing on an independent sample was then used to validate this analysis. A model was established with a sensitivity of $98 \%$ and specificity of $95 \%$ for the learning sample set, and a sensitivity of $96 \%$ and a specificity of $72 \%$ for the validation dataset. Initial protein database searching identified four peptides of model variable importance, namely S100-A6, S100-P, S100-A9 and S100-A12, which are all small $\mathrm{Ca}^{2+}$ binding proteins that participate in many cellular functions including tumor growth [1]. Individually, these molecules had been previously demonstrated to be potential biomarkers in diverse disease processes, including cancer of the pancreas, bladder, stomach, lung, breast and colon, and also in inflammatory bowel disease [1]. Another class of identified proteins were serine protease inhibitors, namely a fragment of $\alpha$-1-antitrypsin and a fragment of $\alpha$-1-antichymotrypsin, both of which have previously been demonstrated to be markers for lung, prostate and pancreatic cancer [1]. This study recruited a heterogenous group of cancer patients that included individuals with gastric, esophageal, junctional and pancreatic cancer [1]. However, the results highlight clearly the apparent redundancy of biomarkers between different disease processes, and the need to employ a fingerprint of different biomarkers to specify a particular tumor type. The raft of diagnostic biomarkers for upper GI tract cancer that have been described in the literature supports such a conclusion. If we consider the different biofluids in turn, reported biomarkers include:

- Urinary iodine [6], c-erbB-2 [7] and EGF receptor-binding growth factors [8];

- Serum cathepsin [9], CEA, CA19-9, TFF3 [10], tumor pyruvate kinase M2-PK [11] and tumor antigen REG4 [12];

- Fecal DNA methylation [13], CEA and glycoprotein 87 [14];

- Salivary proteomic fingerprint analysis [15], plus many others.

The ${ }^{13} \mathrm{C}$-uracil breath test has also been used to predict the anticancer effect of oral S-1 administration in gastric cancer [16], whereas the etiological link between Helicobacter pylori 
infection and gastric cancer suggests a potential role for the urea breath test in gastric cancer risk prediction. From a genetics perspective, there are also several hereditary causes of upper GI tract cancer, most of which are inherited in an autosomal dominant fashion and can be identified by a specific germline genetic marker. For example, esophageal cancer can occur in several syndromes associated with inherited tylosis (keratoderma of the palms and soles), namely diffuse nonepidermolytic palmoplantar keratoderma and Howel-Evans syndrome (mutation at locus $17 \mathrm{q} 25$ ). Examples of inherited conditions associated with gastric cancer include hereditary diffuse gastric cancer ( $C D H 1$ mutation), Li-Fraumeni syndrome ( $p 53$ mutation), Lynch syndrome (also known as hereditary nonpolyposis colorectal cancer, caused by mutation in one of several DNA mismatch repair genes); familial adenomatous polyposis (APC mutation); Peutz-Jeghers syndrome (STK11 mutation); Cowden syndrome (PTEN mutation); inherited breast cancer (BRCA1 and BRCA2 mutations); and Carney-Stratakis syndrome ( $S D H B, S D H C$, and $S D H D$ mutations). For sporadic upper GI tract cancers, there is now also a long list of SNPs (some of which involve those genes also involved in germline mutations) that have been associated with both risk of development of cancer and risk of recurrence [17].

Beyond disease diagnosis, much of our laboratory's previous work has focused on the role of biomarkers in the prediction of prognosis in upper GI tract cancer, particularly with reference to the syndrome of inappropriate nutritional depletion called cachexia. We have employed MALDI-TOF MS analysis to demonstrate that urine can be used as a matrix in which to identify biomarkers of cachexia, including the breakdown products of skeletal muscle [2]. Furthermore, over the last 20 years, we have investigated a number of blood-based biomarkers of poor prognosis in upper GI cancer, including C-reactive protein (CRP), pro-inflammatory cy tokines, parathyroid hormone-related peptide [18], and macrophage inhibitory cytokine-1 [19]. Many cancer patients have abnormal circulating concentrations of these markers even in the absence of cachexia, which proves that these markers also have a diagnostic quality. However, once again, most of these detected abnormalities have been reproduced in isolation in other tumor types, re-emphasising the need for composite biomarker fingerprints. The analysis of these fingerprints may also offer added information on disease stratification. For example, in male pancreatic cancer patients, patients with hypogonadism (low testosterone) and elevated circulating CRP exhibit worsened survival compared with patients with hypogonadism or elevated CRP alone [20]. Genetic analysis may also be used to determine biomarkers for prognosis. Previous studies by our group have identified SNPs in inflammatory cytokine genes [18] that influence survival of gastro-esophageal cancer patients.

In summary, the evidence would suggest that many biomarkers of upper GI tract cancer have already been described. However, the next step is to combine these markers into composite fingerprints that identify individual tumor types, possibly by analysing different biofluids simultaneously. Following this step, the next hurdle will be to develop a simple, clinical method of fingerprint assay that is sensitive, reproducible and cost effective (e.g., a urine dipstick test), but this ease of technology currently remains elusive. It is also difficult to imagine a time when noninvasive biomarker analysis will replace formal histopathological diagnosis. Thus, the ultimate use of diagnostic biomarkers may be as a clinical screening tool rather than as a strict confirmation of positive diagnosis. Patients would be asked to complete noninvasive testing for upper GI tract cancer, and those with a positive test would be invited to upper GI tract endoscopy (in an analogous fashion to the way in which fecal occult blood testing and colonoscopy are used during bowel cancer screening).

Undoubtedly, the research will continue and will produce exciting results, but a final biomarker fingerprint for upper GI tract cancer seems several years away. Perhaps the quest for biomarkers will be achieved through different means altogether. As methods of radiological imaging (including 'virtual' endoscopy and functional imaging) become more sensitive and less toxic, it is not inconceivable that the ultimate noninvasive method of all cancer diagnosis will soon be a whole-body scan.

Financial \& competing interests disclosure

The authors have no relevant affliations or financial involvement with any organization or entity with a financial interest in or financial conflict with the subject matter or materials discussed in the manuscript. This includes employment, consultancies, honoraria, stock ownership or options, expert testimony, grants or patents received or pending, or royalties.

No writing assistance was utilized in the production of this manuscript. 


\section{Bibliography}

1. Husi H, Stephens N, Cronshaw A et al. Proteomic analysis of urinary upper gastrointestinal cancer markers. Proteomics Clin. Appl. 5(5-6), 289-299 (2011).

2. Skipworth RJ, Stewart GD, Bhana M et al. Mass spectrometric detection of candidate protein biomarkers of cancer cachexia in human urine. Int. J. Oncol. 36(4), 973-982 (2010).

3. Mischak H, Allmaier G, Apweiler R et al. Recommendations for biomarker identification and qualification in clinical proteomics. Sci. Transl Med. 2(46), 46ps42 (2010).

4. Adachi J, Kumar C, Zhang Y, Olsen JV, Mann M. The human urinary proteome contains more than 1500 proteins, including a large proportion of membrane proteins. Genome Biol. 7(9), R80 (2006).

5. Alterovitz G, Xiang M, Liu J, Chang A, Ramoni MF. System-wide peripheral biomarker discovery using information theory. Pac. Symp. Biocomput. 231-242 (2008).

6. Gulaboglu M, Yildiz L, Gul M, Celebi F, Peker K. Blood and urine iodine levels in patients with gastric cancer. Biol Trace Elem. Res. 113(3), 261-271 (2006).

7. Tsigris C, Karayiannakis AJ, Syrigos KN et al. Clinical significance of soluble c-ErbB-2 levels in the serum and urine of patients with gastric cancer. Anticancer Res. 22(5), 3061-3065 (2002).

8. Chuang LY, You HL, Tsai JF. Epidermal growth factor receptor-binding growth factors in the urine of patients with cancers of the digestive tract. J. Gastroenterol. 35(8), 598-606 (2000).
9. Szajda SD, Snarska J, RoszkowskaJakimiec W et al. Activity of cathepsin D in the blood serum and urine of patients with cancer of the stomach, pancreas and liver. Pol. Arch. Med. Wewn. 116(6), 1150-1154 (2006).

10. Aikou $S$, Ohmoto Y, Gunji T et al. Tests for serum levels of trefoil factor family proteins can improve gastric cancer screening. Gastroenterology 141(3), 837-845 (2011).

11. Hathurusinghe HR, Goonetilleke KS, Siriwardena AK. Current status of tumor M2 pyruvate kinase (tumor M2-PK) as a biomarker of gastrointestinal malignancy. Ann. Surg. Oncol. 14(10), 2714-2720 (2007).

12. Kobayashi Y, Niwa Y, Tajika M et al. Serum tumor antigen REG4 as a useful diagnostic biomarker in gastric cancer. Hepatogastroenterology 57(104), 1631-1634 (2010).

13. Nagasaka T, Tanaka N, Cullings HM et al. Analysis of fecal DNA methylation to detect gastrointestinal neoplasia. J. Natl Cancer Inst. 101(18), 1244-1258 (2009).

14. Qiao SX, Yuan M, Liu YL, Lin XS, Zhang XP, Tobi M. Detection of gastric cancer and premalignant lesions by novel marker glycoprotein 87 using monoclonal antibody Adnab-9. Cancer Epidemiol. Biomarkers Prev. 12(10), 1095-1099 (2003).

15. Wu ZZ, Wang JG, Zhang XL. Diagnostic model of saliva protein finger print analysis of patients with gastric cancer. World J. Gastroenterol. 15(7), 865-870 (2009).

16. Ishii Y, Suzuki S, Takahashi Y, Takayama T, Asai S. Can the 2-(13)C-uracil breath test be used to predict the effect of the antitumor drug S-1? Cancer Chemother. Pharmacol. 66(2), 333-343 (2010).
17. Persson C, Canedo P, Machado JC, El-Omar EM, Forman D. Polymorphisms in inflammatory response genes and their association with gastric cancer: a HuGE systematic review and meta-analyzes. Am. J. Epidemiol. 173(3), 259-270 (2011).

18. Stephens NA, Skipworth RJ, Fearon KC. Cachexia, survival and the acute phase response. Curr. Opin Support. Palliat. Care 2(4), 267-274 (2008).

19. Skipworth RJ, Deans DA, Tan BH et al. Plasma MIC-1 correlates with systemic inflammation but is not an independent determinant of nutritional status or survival in oesophago-gastric cancer. Br. J. Cancer 102(4), 665-672 (2010).

20. Del Fabbro E, Hui D, Nooruddin ZI et al. Associations among hypogonadism, C-reactive protein, symptom burden, and survival in male cancer patients with cachexia: a preliminary report. J. Pain Symptom Manage. 39 (6), 1016-1024 (2010).

\section{Website}

101. Office of National Statistics Cancer Survival: England and Wales, 1991-2001, twenty major cancers by age group (2007) www.ons.gov.uk/ons/publications/ re-reference-tables. html?edition $=\mathrm{tcm} \% 3 \mathrm{~A} 77-51080$ 